\title{
Influência da publicidade na automedicação na população de um município brasileiro de médio porte
}

\section{Influence of advertising in self-medication among the population of a brazilian midsize town}

Jucier Gonçalves Júnior ${ }^{1}$, Sávio Emanuel dos Santos Moura ${ }^{1}$, Gabriela Carvalho Lage Dantas ${ }^{1}$, Antônia Máximo de Lima ${ }^{1}$, Wânia Sandra Bezerra de Brito ${ }^{2}$, Bárbara de Oliveira Brito Siebra ${ }^{1}$, Jair Paulino de Sales ${ }^{3}$, Estelita Lima Cândido ${ }^{1}$

1. Discente do curso de medicina pela Universidade Federal do Cariri (UFCA), Crato, CE, Brasil. 2. Laboratório Central de Saúde Pública do Ceará (LACEN), Crato, CE, Brasil. 3. Mestrando do Programa de Desenvolvimento Regional pela Universidade Federal do Cariri (UFCA), Crato, CE, Brasil. 4. Docente do curso de medicina pela Universidade Federal do Cariri (UFCA), Crato, CE, Brasil.

\section{Resumo}

Introdução: A automedicação é uma prática que, feita de forma arbitrária, traz riscos reais à saúde da população. Entretanto, seus fatores contributivos, sobretudo a influência da propaganda no consumo de fármacos, não tem uma correlação bem estabelecida na literatura. Objetivos: Estimar a prevalência da automedicação e avaliar a influência da propaganda nesse hábito. Método: estudo transversal, quantitativo, no município de Crato, Ceará, Brasil, com amostra de 104 pessoas. Resultados: $67 \%$ da amostra praticava automedicação. Destas, $80 \%$ conheciam os riscos para a prática. Uma parcela de $67,6 \%$ dos praticantes revelou ser influenciada pela propaganda para a escolha do medicamento e, entre eles, a taxa de automedicação foi de 1,6x maior ( $\mathrm{p}=0,004$ ). 0 sexo, a idade e a renda não exerceram influência sobre a referida prática $(p>0,05)$. Conclusões: A prevalência de automedicação entre os participantes é elevada, o que denota uma necessidade real de se repensar as normas regimentais de publicidade, assim como desperta e sugere para o impacto que campanhas publicitárias bem elaboradas podem ter no público em geral, configurando uma potencial ferramenta de saúde pública.

Palavras-chave: Automedicação. Saúde Pública. Propaganda.

\begin{abstract}
Introduction: DSelf-medication is a practice that, when carried out in an arbitrary way, brings real risks to the health of the population. However, its contributory factors, especially the influence of advertising on drug consumption, do not have a well-established correlation. Objectives: To estimate the prevalence of self-medication and to evaluate the influence of advertising on such habit. Method: A cross-sectional and quantitative study was carried out in the city of Crato, Ceará, Brazil, with a sample of 104 people. Results: It was evidenced that $67 \%$ of the sample practiced self-medication, of which $80 \%$ knew the risks to practice. A portion of $67.6 \%$ of practitioners was shown to be influenced by the advertising for drug choice, and among these the selfmedication rate was $1.6 x$ higher $(p=0.004)$. Sex, age and income exerted no influence the practice $(p>0.05)$. Conclusion: The prevalence of self-medication among the participants is high, which indicates a real need to rethink the regimental norms of publicity, as well as awakens and suggests that the impact of well-designed advertising campaigns can have on the general public, making up a potential public health tool.
\end{abstract}

Keywords: Self-medication. Public health. Advertising.

\section{INTRODUÇÃO}

A automedicação é uma forma de autocuidado à saúde definida como a obtenção ou o consumo de medicamentos sem consulta de um médico, seja para diagnóstico, prescrição ou vigilância do tratamento ${ }^{1,2}$. Em muitos casos, os medicamentos são fornecidos por outras pessoas, geralmente parentes ou amigos, que possuem tanto a medicação como o conhecimento sobre seu uso, ou emprestam uma prescrição antiga para a compra de um novo medicamento sem a consulta médica a fim de saber se aquele princípio ativo é apropriado para a enfermidade ${ }^{3}$.

A Organização Mundial de Saúde (OMS) recomenda a automedicação como um meio de desonerar o sistema público de saúde, desde que seja acompanhada por orientação adequada ao consumidor ${ }^{4}$. Entretanto, essa prática nem sempre é segura, uma vez que pode estar relacionada com o autodiagnóstico incorreto, os atrasos em busca por consultas médicas, as reações adversas, as interações medicamentosas perigosas, a dosagem incorreta, o erro na escolha da terapia, o mascaramento de uma doença grave e o risco de dependência e abuso ${ }^{5,6}$.

Na sociedade moderna, à medida que o acesso ao consumo foi convertido no objetivo principal para o desfrute de níveis satisfatórios de bem-estar, bons níveis de saúde passaram a ser vistos como possíveis na estreita dependência do acesso a tecnologias diagnóstico-terapêuticas. "O consumo é algo inerente ao homem", havendo uma relação entre as transformações da sociedade e o fenômeno do consumo, embora o medicamento não esteja desvinculado dessa característica social ${ }^{7,8}$. 
Mesmo que a Política Nacional de Medicamentos, no Brasil, dê especial ênfase ao processo educativo dos usuários ou consumidores acerca dos riscos da automedicação, da interrupção e da troca da medicação prescrita, o consumo de medicamentos por conta própria pode ser influenciado negativamente pelo acesso sem barreiras e pela promoção e publicidade de remédios, que, muitas vezes, estimulam a utilização desnecessária e irracional ${ }^{9,10}$.

Os jovens representam uma população vulnerável aos meios de comunicação, principalmente à internet, que apresentam muitas publicidades estimulando o consumo, inclusive de medicamentos ${ }^{11}$. Nesse contexto, o objetivo deste estudo foi determinar a prevalência de automedicação na população jovem de um município de médio porte do Nordeste brasileiro e descrever a influência da propaganda nessa prática.

\section{MÉTODOS}

Pesquisa transversal e quantitativa realizada no município de Crato, cidade sul cearense de médio porte considerada polo comercial, cultural e educacional, o que aumenta as chances de seleção de participantes pertencentes às faixas etárias, foco desta pesquisa, jovens e adultos. Uma amostra foi calculada por meio do programa Epi Info versão 3.5.1 considerando a população de adultos jovens igual a 42.623 (estimativa para 2013 com base no censo 2010) ${ }^{12}$, para o referido município, e uma taxa de prevalência de automedicação equivalente a $90 \%$ \pm 513 . Assim, com $95 \%$ de confiança, foi estimado um número de 138 pessoas para compor a amostra. Porém, os dados de 34 participantes foram excluídos da presente análise por possuírem idade abaixo de 18 anos, o que necessitaria do consentimento do seu responsável.

A escolha dos participantes da pesquisa foi feita de forma não aleatória, tendo sido realizada no Centro da cidade, já que este bairro comporta grande fluxo de moradores locais, obtendo, assim, uma cobertura maior da população municipal.

A coleta de dados se deu durante os anos de 2013 - 2014, por meio de um questionário, contendo 38 questões, aplicado pelos próprios pesquisadores de modo presencial. 0 instrumento abordava características socioeconômicas, automedicação e publicidade. Sobre este último ponto, foi selecionado, de modo não aleatório, os sete slogans publicitários mais veiculados no período, para que os participantes identificassem os fármacos correspondentes.

Os dados foram tabulados e submetidos à análise estatística por meio do programa Epi-Info, versão 3.5.1. Inicialmente, foi realizada uma análise univariada para a determinação de prevalências, medidas de tendência central e de variabilidade; em seguida, a análise bivariada, a qual permitiu calcular medidas de associação, entre elas, a razão de prevalência, bem como testar a significância estatística das associações, considerando um nível de 0,05.

De acordo com a Resolução no 466/2012, os aspectos éticos foram respeitados, sendo esclarecido o objetivo do estudo aos participantes. A autorização para a aplicação dos questionários com a população foi obtida por meio da assinatura do Termo de Consentimento Livre e Esclarecido pelos entrevistados, no qual constaram os objetivos da pesquisa, seu caráter voluntário e sigiloso além do contato do pesquisador e do orientador para eventuais esclarecimentos. Esta pesquisa foi aprovada pelo Comitê de Ética em Pesquisa com Seres humanos da Faculdade de Medicina da Universidade Federal do Cariri - UFCA sob o parecer 20683913.7.0000.5035.

\section{RESULTADOS}

Os 104 entrevistados apresentaram predominância do sexo feminino $(65,3 \%)$, com ensino superior incompleto (53\%) e renda $\leq 1-2$ salários mínimos $(85,7 \%)$. A idade dos participantes do estudo variou de 18 a 41 anos (média=23 23,4 ). A minoria $(28,85 \%)$ se declarou como chefe de família, e destes, cerca de $32 \%$ tinham filhos que moravam com eles.

A prevalência de automedicação no grupo foi de $67,65 \%$. Com exceção de um caso, todos afirmaram automedicar-se motivados pela dor, destacando-se como queixas principais a cefaleia (88) e a febre (59); a dor na garganta (55) e as cólicas (43). Aproximadamente $88 \%$ dos respondentes afirmaram que se automedicavam porque já haviam utilizado o medicamento antes; 34,7\% devido à praticidade e, em menor proporção, a prática era realizada graças à dificuldade de acesso ao serviço de saúde, ou por considerarem que a doença a ser tratada não era grave. Aproximadamente $80 \%$ deles afirmaram conhecer os riscos que a prática proporciona.

Uma parcela de $41 \%$ dos entrevistados afirmou ter tido acesso a alguma propaganda de remédio recentemente. Os meios de comunicação predominantes foram internet $(72,1 \%)$ e televisão $(56,7 \%)$. Esses meios são acessados pela maioria, diariamente, por períodos de duas horas ou menos $(41,7 \%)$ ou de $3-5$ horas por dia (26,2\%).

Os medicamentos citados foram analgésicos, antitérmicos, fármacos para desconfortos gástricos, antibiótico e antigripal. Uma proporção de $67,6 \%$ de todos os entrevistados acreditava que a propaganda havia influenciado a escolha destes medicamentos.

Dos sete slogans publicitários apresentados aos participantes, em apenas uma o fármaco correspondente foi citado corretamente por todos os participantes. Trata-se do analgésico Benegripe $^{\circledR}$, cujo uso foi referido por cerca de $63 \%$ dos que reconheceram seu slogan (Tabela 1 ).

O total de acertos avalia a eficiência de uma propaganda, mas os erros indicam que, assim como reconheceram erroneamente um medicamento e referiram seu uso, sua autoindicação bem como sua posologia podem ter ocorrido equivocadamente.

Os outros slogans reconhecidos corretamente por $90 \%$ ou mais foram os correspondentes aos fármacos Doril ${ }^{\circledR}(97,4 \%)$ 
e Neosaldina ${ }^{\circledR}(90,2 \%)$, sendo consumidos por $31,1 \%$ e $53,6 \%$, respectivamente (Tabela 1 ).

Tabela 1. Reconhecimento dos slogans de propagandas medicamentosas e automedicação.

\begin{tabular}{lrrr}
\hline Slogan* & Reconheceram & Acertaram & Usaram \\
\hline $\begin{array}{l}\text { "Tomou Doril, a dor } \\
\text { sumiu" }\end{array}$ & 77 & 75 & 24 \\
"É gripe? Benegripe" & 81 & 81 & 51 \\
"Eno é alívio já" & 31 & 14 & 16 \\
"Dor de cabeça? Chama a & & & \\
Neosa (Idina)" & 41 & 37 & 22 \\
"Tira dor, desentope & & & \\
e revigora: Coristina D" & 13 & 10 & 8 \\
"Passa Gelol que passa" & 41 & 23 & 29 \\
"Atroveran: tomou, & 36 & 19 & 17 \\
passou" & & & \\
"O nome do medicamento foi omitido na hora da pergunta. &
\end{tabular}

Ao investigarmos a relação entre características pessoais e automedicação, apenas o acesso à propaganda apresentou associação estatisticamente significante $(p=0,004)$. Entre os entrevistados que responderam positivamente à pergunta, a prática de automedicação foi 1,6 vezes maior.

A prevalência de automedicação foi maior no grupo feminino $(68,66 \%)$, mas a diferença não foi suficientemente grande para indicar significância $(p=0,76)$.

A idade não influenciou a automedicação, já que a média de idade do grupo que se automedicava foi a mesma do a que respondeu negativamente (média=23; $p=0,84$ ). Do mesmo modo, a renda não exerceu qualquer influência no processo de automedicação $(p=0,14)$.

\section{DISCUSSÃO}

Quarenta e um por cento dos entrevistados disseram ter tido acesso a alguma propaganda de remédio recentemente, e $67,6 \%$ reconheceram que a prática de se automedicar estava relacionada às propagandas publicitárias. Os meios de comunicação de massa, como televisão, jornais, revistas e internet, são veículos utilizados pela indústria farmacêutica que visam atrair o máximo de compradores para seus produtos ${ }^{14}$. Essa relação também foi observada em outros estudos: Barbosa e Boechat ${ }^{15}$ e Silva et. $a^{16}$.

A propaganda foi veiculada, predominantemente, pela internet $(72,1 \%)$ e pela televisão $(56,7 \%)$. O acesso a esses meios se dá diariamente, por um período $\leq 2$ horas $(41,7 \%)$ ou de $3-5$ horas por dia (26,2\%). A influência das mesmas mídias na automedicação também foi constatada em estudo realizado em Fortaleza - CE, com 722 estudantes de escolas públicas e privadas, embora nesse estudo a televisão tenha ocupado a primeira posição $(93,1 \%)^{16}$. A esse respeito, Thiago, Russo e
Camargo $\mathrm{Jr}^{14}$ consideram a internet um importante dispositivo de produção e divulgação de ideias, conceitos e informações na área de saúde e um recurso a serviço da divulgação científica.

Quanto aos sete slogans de propagandas apresentados aos participantes, apenas o referente ao Benegripe ${ }^{\circledR}$ foi citado corretamente por todos os participantes e consumido por $63 \%$ dos que reconheceram seu slogan. Os outros reconhecidos corretamente por $90 \%$ ou mais foram os correspondentes aos fármacos Doril $^{\circledR}(97,4 \%)$ e Neosaldina ${ }^{\circledR}(90,2 \%)$, sendo consumidos por $31,1 \%$ e $53,6 \%$, respectivamente.

O nome fantasia de um medicamento é dotado de um valor significativo para a empresa e, por isso, seu registro é protegido internacionalmente. Os teóricos do Marketing relatam casos em que alguns nomes fantasia alcançam valores maiores no mercado de ações que os do parque industrial instalado na indústria que os produziram ${ }^{17}$. Para Alves e Whitaker ${ }^{18}$, os publicitários criam propagandas que exaltam valores culturais significativos para nossa sociedade e dosam certa carga de ideologia, buscando estabelecer um vínculo emocional, ainda que inconsciente, entre a mercadoria divulgada e o indivíduo consumidor.

Ao se investigar a relação entre características pessoais e automedicação, somente o acesso à propaganda obteve associação estatisticamente significante $(p=0,004)$, embora entre os entrevistados que responderam positivamente à pergunta, a automedicação tenha sido 1,6 vezes maior. Para Galato et. al $^{19}$, a propaganda exerceu significante influência sobre a automedicação $(p<0,001)$ no grupo de universitários estudado, sobretudo naqueles que não eram da área de saúde.

Muitos estudos, dentre eles os supracitados, confirmam o poder da propaganda como estimulante da automedicação ${ }^{18}$. Por meio dela, o indivíduo assimila informação sobre as indicações dos medicamentos que podem ser adquiridos na farmácia sem receita médica. Muitas vezes, o medicamento utilizado pelo consumidor não era indicado para sua enfermidade, podendo contribuir para o agravamento do quadro mórbido. Segundo a OMS, mais de $50 \%$ de todos os medicamentos são incorretamente prescritos, dispensados e vendidos, e metade dos pacientes utiliza de maneira errada ${ }^{20,21}$.

Além disso, Rios et. al ${ }^{22}$ afirmam que a propaganda desenfreada e massiva de determinados medicamentos contrasta com as tímidas campanhas que tentam esclarecer os perigos da automedicação. Em 2011, os medicamentos corresponderam a $29,5 \%$ dos casos de intoxicações registrados no Brasil e a $16,9 \%$ dos casos de óbito por intoxicações ${ }^{21}$.

Essa realidade se confirma ao destacar que, em 2007, no Brasil, cerca de R\$ 863 milhões foram gastos com publicidade no setor dos medicamentos isentos de prescrição (MIP), distribuídos entre os seguintes veículos: 75\% para televisão (canal aberto), $14 \%$ para rádio, $5 \%$ para televisão por assinatura, $5 \%$ em revistas e $1 \%$ para jornais ${ }^{23}$. 


\section{CONCLUSÃO}

Constatou-se que a propaganda exerce forte influência sobre a automedicação, e que muitos usuários podem ter-se equivocado na realização desta prática, do mesmo modo que confundiram os slogans dos fármacos e afirmaram seu uso. A automedicação adotada erroneamente acarreta consequências extremamente danosas à saúde da população, como aumento da resistência aos antimicrobianos, efeitos colaterais, ações teratogênicas e interações medicamentosas fatais, que, embora sejam previstas por profissional qualificado, são desconhecidas pelo grande público. Dessa forma, a automedicação, embora estimulada por políticas de saúde, deve ser monitorada e acompanhada de constantes medidas socioeducativas. Sugere-se, ainda, que haja mais rigor na fiscalização do comércio de medicamentos no País.

\section{REFERÊNCIAS}

1. Oliveira MA, Francisco PMSB, Costa KS, Barros MBA. Self-medication in the elderly population of Campinas, São Paulo state, Brazil: prevalence and associated factors. Cad. Saúde Pública. 2012 Fev; 28(2): 335-45. doi:http:// dx.doi.org/10.1590/S0102-311X2012000200012.

2. Paim RSP, Lunelli RP, Zanchett K, Menon P, Costa S, Giachelin T. Automedicação: uma síntese das publicações nacionais. Revista Contexto \& Saúde. 2016 JanJun; 16(30): 47-54. doi: http://dx.doi.org/10.21527/2176-7114.2016.30.47-54

3. Gualano MR, Bert F, Passi S, Stillo M, Galis V, Manzoli L, et al. Use of selfmedication among adolescents: a systematic review and meta-analysis. Eur J Public Health; 2015 Jun; 25(3): 444-50. doi: 10.1093/eurpub/cku207.

4. Conselho Regional de Farmácia do Estado de São Paulo (CRF-SP). Farmácia não é um simples comércio. Fascículo II, 2010.

5. Ribeiro DI Jr, Santos AF. Portal Semântico sobre automedicação: um instrumento para prevenção e orientação à comunidade. RBBD. 2016; 11(esp): 391-410.

6. Bertoldi AD, Camargo AL, Silveira MP, Menezes AM, Assunção MC, Gonçalves $\mathrm{H}$, et al. Self-medication among adolescents aged 18 years: the 1993 Pelotas (Brazil) birth cohort study. J Adolesc Health. 2014 Aug; 55(2): 175-181. doi: 10.1016/j.jadohealth.2014.02.010. PubMed PMID: 24713443.

7. Carvalho MMB. Manipulação das preferências de consumo: alienação humana e degradação ambiental nos caminhos de um modelo social insustentável. Revista de Direito, Glob e Res. nas Rel. de Cons. 2015 Jul-Dez; 1(2): 167-190. doi: http://dx.doi.org/10.21902/2526-0030/2015.v1i2.91.

8. Aquino DS, Barros JAC, Silva MDP. Self-medication and health academic staff. Ciênc. saúde coletiva. 2010 Ago; 15(5): 2533-8. doi: http://dx.doi.org/10.1590/ S1413-81232010000500027.

9. Ministério da Saúde (BR). Secretaria de Políticas de Saúde. Departamento de Atenção Básica. Política Nacional de Medicamentos. Brasília: Ministério da Saúde; 2001. 39p.

10. Naves JOS, Castro LLC, Carvalho CMS, Merchan-Hamann E. Automedicação: uma abordagem qualitativa de suas motivações. Cad saúde coletiva. 2010 Jun; 15(supl1): 1751-62. doi: http://dx.doi.org/10.1590/S141381232010000700087.

11. Kumar N, Kanchan T, Unnikrishnan B, Rekha T, Mithra P, Kulkarni V et al. Perceptions And practices of self-medication among medical students in Coastal South India. Plos One. 2013 Aug; 8(8): E72247. doi: https://doi.org/10.1371/ journal.pone.0072247.

12. Instituto Brasileiro de Geografia e estatística. Estimativas populacionais para os municípios brasileiros em 01.07.2013 [Internet]. Rio de Janeiro: IBGE; 2013 [acesso 2013 Out 15]. Disponível em: https://ww2.ibge.gov.br/home/ estatistica/populacao/estimativa2013/default.sht.

13. Instituto de Ciência, Tecnologia e Qualidade. Automedicação no Brasil. [Internet]. São Paulo: ICQT; 2014 [acesso 2014 Abr 30]. Disponível em: http:// www.ictq.com.br/pesquisa-do-ictq/353-indicacao-de-amigo-reforca-a-praticada-automedicaca.

14. Thiago CC, Russo JA, Camargo Júnior KR. Hormônios, sexualidade e envelhecimento masculino: um estudo de imagens em websites. Interface. 2016 Jan-Mar; 20(56): 37-50. doi: http://dx.doi.org/10.1590/1807-57622014.0031.

15. Barbosa LB, Boechat MSB. Perfil da automedicação em estudantes do município de Laranjal-MG. Acta Biomédica Brasiliensia. 2012 Jan; 3(1): 98-109.

16. Silva IM, Catrib AM, de Matos VC, Gondim AP. Self-medication in adolescence: a challenge to health education. Ciênc. Saude Colet. 2011; 16(1): 1651-1660. PubMed PMID: 21503517.

17. Nascimento AC. Propaganda de medicamentos para grande público: parâmetros conceituais de uma prática produtora de risco. Ciênc. saúde coletiva. 2010 Nov; 15(supl 3): 3423-32. doi: http://dx.doi.org/10.1590/S141381232010000900017.

18. Alves FCR, Whitaker DCA. A relação da televisão e da internet com o consumo: análises da ideologia e da cultura nas propagandas. ReBraM. 2016 Jul; 19(1): 55-62. doi: https://doi.org/10.25061/2527-2675/ReBraM/2016. v19i1.374.

19. Galato D; Madalena J; Pereira GB. Self-medication among university students: the influence of the field of study. Ciênc. Saude Colet. 2012 Dec; 17(12): 3323-30. PubMed PMID: 23175408.

20. Corrêa AD, Caminha JR, de Souza CA, Alves LA. Uma abordagem sobre o uso de medicamentos nos livros didáticos de biologia como estratégia de promoção de saúde. Ciênc. saúde coletiva. 2013 Out; 18(10): 3071-82. doi: http://dx.doi. org/10.1590/S1413-81232013001000032.

21. Domingues PHF, Galvão TF, Andrade KRC, de Sá PTT, Silva MT, Pereira MG. Prevalence of self-medication in the adult population of Brazil: a systematic review. Rev. Saude Publica. 2015; 49:1-36. doi: 10.1590/S00348910.2015049005709. PubMed Central PMCID: PMC4544343.

22. Rios MF, Souza WA, Siqueira VMS, Podestá MHMC, Melo GGP, Zuba AG, et al. Perfil da automedicação dos alunos de uma escola técnica do Sul de Minas Gerais. Rev. Unincor. 2013; 11(2): 420-1. doi: http://dx.doi.org/10.5892/ruvrd. v11i2.420431.

23. Batista, AM, Carvalho, MCRD. Avaliação da propaganda de medicamentos veiculada em emissoras de rádio. Ciênc. saúde coletiva. 2013 Fev; 18(2): 553561. doi: http://dx.doi.org/10.1590/S1413-81232013000200027.

\section{Como citar este artigo/How to cite this article:}

Gonçalves J Júnior, Moura SES, Dantas GCL, Lima Am, Brito WSB, Siebra BOB, et al. Influência da publicidade na automedicação na população de um município brasileiro de médio porte. J Health Biol Sci. 2018 Abr-Jun; 6(2):152-155. 